\title{
Agresividad impulsiva y proactiva, moldes mentales y rasgos de personalidad en adolescentes.
}

Impulsive and proactive aggression, mental molds and personality traits in adolescents.

\author{
Ángela Socastro-Gómez ${ }^{1}$ \\ Ana Jiménez-Perianes ${ }^{2}$
}

(1) Universidad Complutense de Madrid, Madrid, España.

(2) Universidad CEU San Pablo, Madrid, España.

Email correspondencia: angelasogo@gmail.com

\section{Resumen}

La agresividad en adolescentes, es un tema de actualidad e interés para la sociedad. La comprensión de las conductas agresivas, en esta etapa del desarrollo, puede mejorar tanto la vida de los adolescentes, como su futuro y el de toda la sociedad. Por todo esto, en el presente trabajo, se estudia la agresividad impulsiva y proactiva que presentan, las diferencias existentes entre los dos sexos, los rasgos de personalidad más característicos de los adolescentes con agresividad y los moldes mentales empleados en sus evaluaciones sobre la realidad que pueden repercutir en el desarrollo de la conducta agresiva. En el presente estudio se han utilizado tres test estandarizados, CAPI-A, 16PF-APQ y MOLDES.

Palabras Clave: Agresividad impulsiva, agresividad proactiva, adolescencia, personalidad, moldes mentales.

\begin{abstract}
Aggression in adolescents is a topic of current interest to society. Understanding aggressive behavior at this stage of development, can improve both the lives of adolescents, as their future and that of the whole society. For all this, in the present work, impulsive and proactive aggression they present, the differences between the sexes, personality traits most characteristic of adolescents with aggression and moldes mentales used in their assessment of reality that can affect the development of aggressive behavior are studied. In the present study we have used three standardized tests, CAPI-A, 16PF-APQ and MOLDES.
\end{abstract}

Keywords: Impulsive aggression, proactive agression, adolescence, personality, moldes mentales.

Fecha de envío: 15/11/2019

Fecha de aceptación: 02/12/2019 


\section{INTRODUCCIÓN}

Existen múltiples definiciones para el término, pero la propuesta por Berkowitz (1996) “disposición relativamente persistente a ser agresivo en diversas situaciones diferentes" (p. 45), es la que más se adapta para la realización de este estudio.

No obstante, Carrasco y Gónzalez (2006) proponen tres puntos en común de todas las definiciones propuestas hasta la fecha, los cuales ayudan a comprender el concepto de agresividad. Como primer punto en común, cabe señalar la intención, así, la conducta agresiva, parece dirigirse siempre hacia una meta concreta, aunque como aportan Dollard, Doob, Miller, Mowrer, y Sears (1939/1968) no siempre denote hacer daño a terceros.

Como segundo punto en común, se hallan las consecuencias negativas asociadas a la conducta agresiva. Estas consecuencias aversivas afectan tanto a la persona agresiva, como a la persona objeto de dicha agresividad.

Como tercer y último punto en común, surge la manifestación heterogénea de la agresividad, es decir, la diversidad de los ataques, tanto físicos como verbales.

\section{A. Manifestaciones de la agresividad}

Según Muñoz Vivas (2000), la agresividad se manifiesta a tres niveles: emocional, cognitivo y conductual. Este último nivel se considera la agresión propiamente dicha, y es consecuencia directa de déficits en destrezas, competencias, habilidades y estrategias de afrontamiento.

Teniendo en cuenta dichos niveles que se ven afectados en la conducta agresiva, Liu (2004), destaca que los tipos de agresión surgen de la interacción entre procesos cognitivos y fisiológicos.

B. Evolución de la agresividad, factores originantes $y$ mantenedores a lo largo del desarrollo

Autores como Dodge y McCourt (2010) y Tremblay et al. (1996) afirman en sus estudios que el origen de la agresividad se encuentra en la infancia temprana, alcanzando su máxima expresión en la adolescencia y disminuyendo en la edad adulta. Para estos autores los niños aprenden a no ser agresivos, pero si el aprendizaje no se desarrolla de manera adecuada, la agresividad aumenta y se cronifica.

Geen (2001) señala como variables internas predisponentes en la agresividad a la personalidad, expectativas, temperamento, observación previa de agresiones y la propia fisiología de la persona. Y, como variables situacionales a las situaciones estresantes en las que se utiliza la agresión como forma de respuesta al incumplimiento de las normas, la frustración o el dolor.

Otros autores como Mestre, Samper, Frías, y Nácher (2003), prefieren centrarse únicamente en variables temperamentales y variables familiares. Las variables internas que señalan son la inestabilidad emocional, la elevada ira-rasgo, la exteriorización de la ira como estrategia de afrontamiento, la falta de control, la falta de autoeficacia o la baja competencia socioemocional. En relación a las variables familiares, la hostilidad y la autonomía entendida como rechazo a atender las necesidades del niño, se convierten en factores precipitantes del comportamiento agresivo.

\section{Tipos de agresividad}

Carrasco y González (2006) señalan que existen múltiples clasificaciones de las conductas agresivas en función de características como la naturaleza de la agresión, la actividad o la relación del agresor con la víctima, entre otros.

Tabla 1. Clasificación y tipos de agresividad

Física Patadas o puñetazos.

Naturaleza Verbal Amenazas o improperios.

Galen y Social, En las relaciones $\begin{array}{ccc}\text { Underwood relacional o } & \text { interpersonales. } \\ \text { (1997) } & \text { simbólica } & \end{array}$

$\begin{array}{lc}\text { Activa } & \begin{array}{c}\text { Compromiso } \\ \text { con la agresión. }\end{array}\end{array}$

Actividad Abilleria

(2012)

Pasiva Falta de respuesta ante la agresión 
víctima-agresor.

$(1992 / 1961)$

Indirecta

(relacional)

Uso de

intermediario

para la agresión.

Producir daño

sin otro

beneficio.

$\begin{array}{ccc}\text { Motivación } & \begin{array}{c}\text { Eerkowitz } \\ (1996)\end{array} & \begin{array}{c}\text { Daño } \\ \text { provocional por } \\ \text { causas internas. }\end{array} \\ & \text { Instrumental } & \begin{array}{c}\text { Daño } \\ \text { provocado para } \\ \text { conseguir algún } \\ \text { beneficio } \\ \text { material. }\end{array} \\ & \end{array}$

Impulsiva

Agresión con provocación.

Fuente de Dodge

instigación (1991)

Proactiva

Agresión, sin provocación, dirigida a un objetivo.

\section{1) Teorías explicativas de la agresividad}

\section{a) La Teoría de la Frustración - Agresión}

Dollard et al. (1939/1968), autores de la teoría, exponen que el uso de la agresión es directamente proporcional a la frustración que tenga la persona. Además, proponen como premisas principales de la misma: (a) la proporción de satisfacción que se ha visto frustrada, (b) el grado en que se impide a la persona alcanzar dicha satisfacción y, (c) la frecuencia de los fracasos previos en la vida de la persona. Así mismo consideran, que los individuos pueden reaccionar de forma no agresiva ante la frustración, y se repara en que el continuo bloqueo de las satisfacciones, incrementa la probabilidad de responder de manera agresiva.

Años más tarde, Berkowitz (1989) aclara que la frustración es un estímulo aversivo que activa la producción de cogniciones automáticas asociadas con la respuesta agresiva, ocasiona afectos negativos, y promueve un aumento de la agresividad.

\section{b) Modelos cognitivos}

El modelo cognitivo social descrito por Dodge y Coie (1987), se basa en el procesamiento de la información social. Los individuos agresivos tienen déficits en el proceso ya que compilan menos información, interpretan la misma con sesgos, generan menos soluciones, eligen la solución menos acertada, y evalúan de manera más positiva las consecuencias de la conducta (Crick \& Dodge, 1996).

En el modelo de procesamiento de la información propuesto por Huesman (1988), la conducta agresiva depende de las capacidades cognitivas y de procesamiento de la información que posean los adolescentes. En la conducta agresiva si el adolescente ha generado "guiones cognitivos" agresivos, estos están más disponibles para la recuperación, aumentando la posibilidad de escoger uno de ellos como patrón conductual.

\section{2) Agresividad y Adolescencia}

Pinillos (1990) indica como factores de riesgo en este estadio a determinados rasgos psicológicos distintivos de esta etapa, la vivencia de una identidad crítica, la imprecisión, la ambigüedad, la fluctuación derivada de la adolescencia en sí misma y la mayor disponibilidad de tiempo del adolescente para centrarse en sí mismo, que tiene como consecuencias un retraso en el proceso de toma de decisiones y condiciona la aparición de comportamientos perturbadores.

Alsaker (1996) focaliza su estudio en los cambios hormonales propios de esta etapa que provocan efectos negativos en el estado de ánimo y en las creencias sobre uno mismo y los demás, que posteriormente se reflejan en la conducta agresiva. Por último, Berkowitz (1996), señala como idea elemental entre la agresividad y la adolescencia, que el aumento de agresividad durante la adolescencia marca la personalidad del individuo, ya que es un período de formación de la misma.

\section{a) Diferencias en función del sexo}

Scandroglio et al. (2002), han demostrado que las mujeres están teniendo más participación y están incrementando el número de conductas violentas, aunque sigue siendo el varón el que más conductas agresivas 
manifiesta. No obstante, otras investigaciones recientes, no observan casi diferencias según el sexo, por ejemplo en relación a la agresividad verbal (Campbell, 2006; Toldos, 2005). Björkqvist, Lagerspetz, y Österman (1994) identifican a los varones como más agresivos, sobre todo, en relación a la agresión directa. Sin embargo, las mujeres utilizan más la agresión relacional o social, también llamada indirecta. En lo concerniente a la agresión reactiva y proactiva, se observan datos similares en ambos sexos, si bien es cierto que la agresión proactiva está más relacionada con el sexo masculino (Bettencourt \& Kernahan, 1997). Sin embargo, parece que las diferencias de género en relación a la agresividad solo se producen durante la infancia (Moffitt \& Caspi, 2001).

\section{3) Personalidad y Agresividad}

López y López (2003), a través de estudios realizados con el HSPQ, es decir, el predecesor del 16PF - APQ, encontraron relaciones entre agresividad, Ansiedad, Independencia, Extraversión y Excitabilidad. Dentro de los factores de primer orden, aparecen correlaciones con inestabilidad emocional, despreocupación por las normas y bajo autocontrol, y también, aunque en menor medida, la culpabilidad. En definitiva, según el 16 PF - APQ, el perfil de una persona con alta agresividad sería, alta impulsividad, atrevimiento y tensión, así como, baja interiorización de normas y autocontrol.

\section{4) Moldes mentales y Agresividad}

Hernández - Guanir (2010) enuncia su teoría sobre los Moldes Mentales. Los moldes mentales son estrategias habituales y características, que muestran los individuos, en el modo de orientar, reaccionar o interpretar la realidad, tanto en situaciones en las que el propio individuo esté implicado, como en situaciones en las que el individuo vea comprometidos sus intereses y emociones. Según el autor, un adolescente agresivo se vería caracterizado por moldes mentales incluidos dentro del encuadre de Ajuste, como Evaluación selectiva negativa, Hostiligencia, Imantación por lo imposible y Desplazamiento emocional. Estos moldes, describirían a un adolescente negativo, hostil, conflictivo, rígido, obsesivo, agresivo e intolerante.

Como conclusión, propone que los adolescentes con alta agresividad poseen un bajo Ajuste y una baja Optimización, como encuadres principales y característicos de su conducta.

\section{MÉtodo}

El objetivo del presente trabajo, consiste en estudiar las posibles diferencias en el marco de la agresividad entre mujeres y hombres adolescentes, describir cuáles son los moldes mentales más característicos de los adolescentes con alta agresividad, así como los rasgos de personalidad más distintivos que explican el desarrollo de dicha conducta agresiva.

\section{A. Muestra}

Los participantes de esta investigación fueron 37 adolescentes (48, $6 \%$ varones y $51,4 \%$ mujeres) con una media de edad de $\mathrm{M}=16,05 ; \mathrm{DT}=0,74$. Todos residentes en la Comunidad de Madrid.

\section{B. Procedimiento}

La participación en esta investigación fue anónima y voluntaria. Las pruebas se administraron en grupos pequeños de 5 a 8 personas, aplicando los tres instrumentos de forma individual y en el mismo día.

\section{Instrumentos}

Uno de los instrumentos utilizados fue el Cuestionario de Agresividad Premeditada e Impulsiva en Adolescentes (CAPI - A; J.M. Andreu, 2010), el cual se basa en la medición de la agresividad proactiva e impulsiva en adolescentes de entre 12 y 17 años de edad.

Otro de los instrumentos aplicados, fue el Cuestionario de Personalidad para Adolescentes 16PF (16PF - APQ; J.M. Schuerger, 2005), que mide 16 rasgos o escalas primarias de personalidad. El cuestionario consta de la escalas primarias de: Afabilidad, Razonamiento, Estabilidad emocional, Dominancia, Animación, Atención a normas, Atrevimiento, Sensibilidad, Vigilancia, Abstracción, Privacidad, Aprensión, Apertura cambio, Autosuficiencia, Perfeccionismo y Tensión.

El último instrumento empleado, fue el Test de estrategias cognitivo - emocionales (MOLDES; P. Hernández - Guanir, 2010), el cual busca conocer los moldes mentales utilizados para identificar, codificar y explicar la realidad en adolescentes y adultos a partir de 14 años. Su estructura se compone de: (a) tres encuadres o factores de tercer orden: Espontaneidad vital, Ajuste y Optimización, (b) diez dimensiones o factores de segundo orden y (c) treinta moldes específicos o factores de primer orden. 


\section{RESUltados}

\section{A. Agresividady sexo de los adolescentes.}

El porcentaje de adolescentes con algún tipo de agresividad en la muestra es de $40,5 \%$, del cual un $73,3 \%$ son mujeres y un $26,7 \%$ son varones. El tipo de agresividad más empleado en la muestra es aquel en el que utilizan la agresividad impulsiva y la proactiva indistintamente $(46,7 \%)$, seguido del tipo Impulsivo $(33,3 \%)$ y por último el tipo Proactivo $(20 \%)$.

Proactiva: se han encontrado diferencias significativas, $\mathrm{t}(35)=-3.16 ; \mathrm{p}<.05$, siendo las mujeres adolescentes las que obtienen una puntuación media mayor $(\mathrm{M}=65.00$; $\mathrm{DT}=21.08)$ en comparación a los varones adolescentes $(\mathrm{M}=41.11 ; \mathrm{DT}=24.76)$.

Impulsiva: se han obtenido diferencias significativas, $\mathrm{t}(35)=-4.72 ; \mathrm{p}<.05$, las mujeres adolescentes alcanzan una puntuación media mayor $(M=72.11 ; D T=20.70)$ en comparación de la alcanzada por los varones adolescentes $(\mathrm{M}=36.11$; $\mathrm{DT}=25.52)$.

\section{B. Moldes mentales y Agresividad Impulsiva.}

Ajuste: se han encontrado diferencias significativas en este encuadre, $\mathrm{t}(35)=-2.71 ; \mathrm{p}<.05$, en la cual, las personas no agresivas $(M=33.6 ; \quad D T=22.70)$ consiguen una puntuación media mayor que los adolescentes de un perfil agresivo impulsivo ( $M=15$; DT=9.04).

Atribución internalista-éxito: los resultados muestran diferencias significativas, $\mathrm{t}(35)=-3.21 ; \mathrm{p}<.05, \quad$ los adolescentes no agresivos logran una puntuación media mayor $(\mathrm{M}=62.64 ; \mathrm{DT}=30.42)$ con respecto a los adolescente agresivos impulsivos $(\mathrm{M}=31.67$; DT=19.46).

Evitación: los resultados significativos, $\mathrm{t}(35)=2.65$; $\mathrm{p}<.05$, alcanzan una puntuación media mayor en los adolescentes agresivos $(\mathrm{M}=89.33$; $\mathrm{DT}=17.16)$, mientras que los adolescentes no agresivos obtienen una media menor $(\mathrm{M}=67.04$; $\mathrm{DT}=26.49)$.

Defensividad: se observan diferencias significativas, $\mathrm{t}(35)=2.85 ; \mathrm{p}<.05$, en la que los adolescentes agresivos tienen una puntuación media mayor $(M=90.25$; $\mathrm{DT}=14.66)$ en contraposición a sus pares no agresivos $(\mathrm{M}=69.04 ; \mathrm{DT}=26.44)$.

Reclusión: se contemplan resultados significativos, $\mathrm{t}(35)=2.03 ; \mathrm{p}<.05$, con una puntuación media mayor $(\mathrm{M}=88.92 ; \mathrm{DT}=10.57)$ de los perfiles agresivos, a la propia de los individuos no agresivos ( $\mathrm{M}=71.28$; $\mathrm{DT}=28.88)$.
Desplazamiento emocional: se han encontrado diferencias significativas $t(35)=2.98 ; \mathrm{p}<.05$, obteniendo puntuaciones medias mayores $(\mathrm{M}=89.42 ; \mathrm{DT}=14.96)$ respecto a sus homólogos no agresivos $(\mathrm{M}=62.64$; $\mathrm{DT}=$ 29.16).

Atribución al temperamento: las diferencias significativas, $\mathrm{t}(35)=2.28 ; \mathrm{p}<.05$, indican puntuaciones medias mayores de los adolescentes agresivos $(\mathrm{M}=78.17$; $\mathrm{DT}=19.72$ ) en comparación a los adolescentes no agresivos $(\mathrm{M}=57.64$; $\mathrm{DT}=27.79)$.

En cuanto al sexo se observaron diferencias significativas en relación a los siguientes moldes.

Espontaneidad vital: las diferencias, $\mathrm{t}(10)=3.35 ; \mathrm{p}<.05$, con una media mayor $(\mathrm{M}=70$; $\mathrm{DT}=.00)$ en los varones, mientras que una puntuación media más baja $(M=29$; DT $=16.63$ ) se relaciona con las mujeres impulsivas.

Implicación vital: los resultados significativos, $\mathrm{t}(10)=.63 ; \mathrm{p}<.05$, señalan puntuaciones medias más altas para los varones $(\mathrm{M}=80 ; \mathrm{DT}=14.14)$ respecto a las mujeres adolescentes $(\mathrm{M}=40 ; \mathrm{DT}=23.57)$.

Hiperanálisis: se obtuvieron diferencias significativas, $\mathrm{t}(10)=-4.17 ; \mathrm{p}<.05$, teniendo menores puntuaciones medias los varones $(\mathrm{M}=25$; $\mathrm{DT}=21.21)$ en comparación a las mujeres $(\mathrm{M}=84.70$; $\mathrm{DT}=18.14)$.

Hipercontrol anticipatorio: otras diferencias significativas son, $\mathrm{t}(10)=-3.12 ; \mathrm{p}<.05$, donde las puntuaciones medias mayores corresponden a las mujeres $(\mathrm{M}=74.70 ; \mathrm{DT}=23.81)$ y las menores a los varones $(\mathrm{M}=20$; $\mathrm{DT}=.00)$.

Afrontamiento borroso: se consiguieron resultados significativos, $\mathrm{t}(10)=-4.55 ; \mathrm{p}<.05$, con puntuaciones medias mayores para las mujeres $(\mathrm{M}=86.50$; $\mathrm{DT}=13.63)$ en comparación a los varones $(\mathrm{M}=35$; $\mathrm{DT}=21.21)$.

Autoconvicción volitiva: este resultado es significativo, $\mathrm{t}(10)=2.48 ; \mathrm{p}<.05$, con puntuaciones medias mayores pertenecientes a los varones $(M=94.50 ; \quad D T=6.36)$ y puntuaciones menores relacionadas con las mujeres $(\mathrm{M}=44 ; \mathrm{DT}=27.56)$.

\section{Moldes mentales y Agresividad proactiva.}

Hiperanálisis: se han encontrado diferencias significativas, $\mathrm{t}(35)=-2.39 ; \mathrm{p}<.05$, obteniendo mayor puntuación media los adolescentes no agresivos $(\mathrm{M}=$ 83.37; $\mathrm{DT}=29.51)$ respecto a los adolescentes agresivos $(\mathrm{M}=57 ; \mathrm{DT}=30.56)$. 
Atribución internalista del éxito: existen diferencias significativas, $\mathrm{t}(35)=-2.08 ; \mathrm{p}<.05$, a favor de las personas no agresivas $(\mathrm{M}=55.74 ; \mathrm{DT}=30.34)$ en relación a sus homólogos con perfil agresivo proactivo $(\mathrm{M}=36$; $\mathrm{DT}=26.75)$.

Espontaneidad vital: se observan diferencias significativas, $\mathrm{t}(35)=2.60 ; \mathrm{p}<.05$, siendo los adolescentes agresivos proactivamente los que tienen una puntuación media mayor $(\mathrm{M}=54.90$; $\mathrm{DT}=29.36)$ en comparación a los adolescentes no agresivos $(\mathrm{M}=30.33$; $\mathrm{DT}=23.98)$.

Implicación vital: las diferencias significativas, $\mathrm{t}(35)=3.537 ; \mathrm{p}<.05$, consisten en puntuaciones medias mayores en los adolescentes agresivos ( $M=25.49$; $\mathrm{DT}=8.06)$ y menores en las personas no agresivas $(\mathrm{M}=24.90 ; \mathrm{DT}=4.79)$.

Implicación directa: se han encontrado resultados significativos, $\mathrm{t}(35)=4.41 ; \mathrm{p}<.05$, con la menor puntuación media de los adolescentes no agresivos ( $M=48.52$; $\mathrm{DT}=24.76)$ a diferencia de los adolescentes agresivos, que obtienen una media mayor $(\mathrm{M}=85.70$; $\mathrm{DT}=15.48)$.

En relación al sexo se observaron diferencias significativas en relación a los siguientes moldes.

Espontaneidad vital: los datos significativos, $t(8)=3.09$; $\mathrm{p}<.05$, señalan puntuaciones medias más altas de los varones $(\mathrm{M}=86.33$; $\mathrm{DT}=14.84)$ en comparación a las mujeres $(\mathrm{M}=41.43$; $\mathrm{DT}=22.67)$.

Implicación vital: los resultados significativos, $\mathrm{t}(8)=2.59 ; \mathrm{p}<.05$, mostrando mayor puntuación media los varones $(\mathrm{M}=90 ; \mathrm{DT}=.00)$ respecto a las mujeres $(\mathrm{M}=54.29 ; \mathrm{DT}=22.99)$.

Hiperanálisis: la diferencia significativa, $\mathrm{t}(8)=-3.30$; $\mathrm{p}<.05$, donde las mujeres obtienen las puntuaciones medias más elevadas $(\mathrm{M}=71.43$; $\mathrm{DT}=22.67)$, mientras que los varones obtienen las puntuación media más baja $(\mathrm{M}=23.33$; $\mathrm{DT}=15.27)$.

Hipercontrol anticipatorio: los resultados significativos, $\mathrm{t}(10)=-2.88 ; \mathrm{p}<.05$, apuntando a puntuaciones medias más altas $(\mathrm{M}=65.71 ; \mathrm{DT}=18.12)$ que corresponden a las mujeres y puntuaciones medias más bajas que corresponden a los hombres $(\mathrm{M}=30$; $\mathrm{DT}=17.32)$.

Afrontamiento borroso: en relación a este molde las diferencias significativas, $\mathrm{t}(10)=-3.66 ; \mathrm{p}<.05$, indican puntuaciones medias mayores en las mujeres $(M=86.57$; $\mathrm{DT}=15.50)$ y puntuaciones medias menores en los varones
$(\mathrm{M}=36.67 ; \mathrm{DT}=28.86)$.

Atribución a las estrategias: los resultados significativos, $\mathrm{t}(8)=-2.49 ; \mathrm{p}<.05$, señalan mayores puntuaciones medias para las mujeres $(\mathrm{M}=59.86$; $\mathrm{DT}=20.49)$ respecto a sus pares varones $(\mathrm{M}=23.33$; $\mathrm{DT}=23.09)$.

\section{Personalidady agresividad impulsiva.}

En los resultados obtenidos se observa que no existen diferencias significativas, en relación al sexo, de los adolescentes con un perfil agresivo impulsivo.

Tensión: las diferencias significativas corresponden a, $\mathrm{t}(35)=2.59 ; \mathrm{p}<.05$, con una media mayor en los adolescentes agresivos $(\mathrm{M}=78.25$; $\mathrm{DT}=17.23)$ respecto a una $(\mathrm{M}=57.12$; $\mathrm{DT}=25.44)$ de los individuos no agresivos.

Ira: las diferencias significativas se observan en, $\mathrm{t}(35)=2.02 ; \mathrm{p}=.05$, la puntuación media mayor corresponde a los individuos agresivos $(\mathrm{M}=88.17$; $\mathrm{DT}=12.96)$ en comparación a la puntuación media de los adolescentes $\sin$ agresividad impulsiva $(\mathrm{M}=74.32$; $\mathrm{DT}=21.84)$.

Dificultades con la autoridad: los resultados significativos, $\mathrm{t}(35)=1.95 ; \mathrm{p}=.05$, incluyen una puntuación media mayor de los adolescentes con perfil de personalidad impulsivo $(\mathrm{M}=78.50$; $\mathrm{DT}=22.92)$ en relación a los demás individuos $(\mathrm{M}=61.16$; $\mathrm{DT}=26.32)$.

Dificultades con la adicción: en cuanto a esta escala las diferencias significativas, $\mathrm{t}(35)=2.55 ; \mathrm{p}<.05$, concluyen puntuaciones medias mayores $(\mathrm{M}=86.83$; $\mathrm{DT}=19.04)$ de los individuos con una agresividad impulsiva alta, a comparación de las puntuaciones medias bajas de los individuos no agresivos $(\mathrm{M}=67$; $\mathrm{DT}=23.36)$.

Total de dificultades: los resultados significativos, $\mathrm{t}(35)=2.38 ; \mathrm{p}<.05$, apuntan a unas puntuaciones medias menores $(M=73.76$; $D T=22.71)$ de los adolescentes no agresivos en confrontación a las personas con alta agresividad ( $\mathrm{M}=91.67$; $\mathrm{DT}=18.04)$.

Afrontamiento deficiente: esta dificultad obtiene diferencias significativas, $\mathrm{t}(35)=2.23 ; \mathrm{p}<.05$, con medias mayores en los individuos con alta agresividad $(\mathrm{M}=81.42$; $\mathrm{DT}=14.33)$ mientras que las puntuaciones medias más bajas $(\mathrm{M}=62.36, \quad \mathrm{DT}=27.73)$ corresponden a los adolescentes sin agresividad impulsiva.

\section{E. Personalidad y agresividad proactiva.}

En los resultados obtenidos se observa que no existen diferencias, en función del sexo, entre los adolescentes con 
altos niveles de agresividad proactiva.

Ira: se han obtenido diferencias significativas, $\mathrm{t}(35)=3.67 ; \mathrm{p}<.05$, donde las puntuaciones medias mayores corresponden a los individuos agresivos proactivamente $(\mathrm{M}=96.20$; $\mathrm{DT}=4.23)$ en comparación a la media de los individuos no agresivos $(\mathrm{M}=72.37$; $\mathrm{DT}=20.14)$.

Dificultades con la autoridad: se han encontrado resultados significativos, $\mathrm{t}(35)=3.17 ; \mathrm{p}<.05$, siendo las puntuaciones medias mayores de los individuos agresivos $(\mathrm{M}=86.90 ; \mathrm{DT}=12.62)$, mientras que las puntuaciones medias más bajas $(\mathrm{M}=59.33$; $\mathrm{DT}=26.22)$ pertenecen a los individuos no agresivos.

Dificultades con la adicción: las diferencias significativas, $\mathrm{t}(35)=2.82 ; \mathrm{p}<.05$, señalan puntuaciones medias mayores de los adolescentes con agresividad proactiva $(\mathrm{M}=90 ; \mathrm{DT}=7.71)$ en relación a los adolescentes no agresivos proactivamente $(\mathrm{M}=67.30$; $\mathrm{DT}=24.79)$.

Total de dificultades: se señalan resultados significativos, $\mathrm{t}(35)=3.39 ; \mathrm{p}<.05$, en los que las puntuaciones de las personas con alta agresividad proactiva son mayores $(\mathrm{M}=97.90$; $\mathrm{DT}=1.59)$ mientras que los adolescentes sin perfil agresivo proactivo obtienen medias menores $(\mathrm{M}=72.78$; $\mathrm{DT}=23.14)$.

\section{F. Tipos de agresividad, desplazamiento emocionaly disociación} emocional.

Se encontraron correlaciones positivas y significativas entre agresividad impulsiva y desplazamiento emocional $(\mathrm{rxx}=, 42, \mathrm{p}<.01)$, a su vez se encontraron correlaciones positivas y significativas entre agresividad proactiva $\mathrm{y}$ disociación emocional $(\mathrm{rxx}=, 42, \mathrm{p}<.01)$.

\section{DISCUSIÓN}

\section{A. Relación entre la agresividady el sexo de los adolescentes.}

A tenor de los datos obtenidos, las mujeres de la muestra presentan más agresividad que los hombres, en contra de lo que exponen autores como Björkqvist et al. (1994), afirmando que los varones adolescentes son más agresivos que las mujeres adolescentes. Esto responde a la tendencia estable de aumento de la agresividad por parte de la población femenina, tal y como mencionaban en su trabajo Scandroglio et al. (2002).

En relación a los dos tipos de agresividad, se observa que las mujeres adolescentes tienen más niveles de agresividad tanto impulsiva como proactiva. Estos datos, no apoyarían lo comentado por Bettencourt y Kernahan (1997), respecto a la no existencia de diferencias en función del sexo en la agresividad impulsiva. Y, por lo tanto, tampoco apoyarían las referencias de estos mismos autores, que señalan como más frecuente, la agresividad proactiva en varones.

\section{B. Relación entre Moldes Mentales y tipos de agresividad.}

La descripción proporcionada por Hernández - Guanir (2010) sobre los moldes mentales implicados en la conducta agresiva, no coincide plenamente con los datos hallados en la muestra. Según el autor, los moldes más significativos en relación a la conducta agresiva, sin distinguir entre impulsiva o proactiva, serían: (a) Evaluación selectiva negativa, (b) Hostiligencia y (c) Desplazamiento emocional. Por lo tanto, el encuadre de Ajuste sería el más relacionado con la conducta agresiva, ya que dos de los moldes mencionados anteriormente lo componen (Evaluación selectiva negativa y Hostiligencia). En los datos hallados y tomando de referencia los significados de los moldes que propone el autor, los adolescentes con una alta agresividad impulsiva tendrían: un bajo Ajuste y una baja Atribución internalista del éxito, lo que supone una mayor inestabilidad en su vida $y$ dificultades para entender que sus cualidades pueden ser el origen de sus éxitos. En consecuencia, poseen una alta Atribución al temperamento, observando sus cualidades internas como causa de sus fracasos. En el polo opuesto, se observa una alta Evitación, Reclusión, Defensividad y Desplazamiento emocional lo que les lleva a escapar de los problemas, sin afrontarlos, siendo propensos a aislarse cuando sufren algún contratiempo, negando o soportando sus fracasos, y prefiriendo liberar sus frustraciones con terceras personas, culpando a los demás.

En relación a las personas con altos niveles de agresividad proactiva, se observa un bajo Hiperanálisis y una baja Atribución internalista del éxito, siendo personas que reflexionan y analizan menos las situaciones de su entorno, y que tienen problemas para ver sus cualidades como la posible causa de sus éxitos. Por el contrario, se observa una mayor Espontaneidad vital, una fuerte Implicación vital y una alta Implicación directa, haciendo que estos adolescentes se comprometan demasiado con todos los acontecimientos que les suceden de una forma impulsiva y vital.

En cuanto a la relación entre la agresividad, el sexo de los adolescentes y los moldes mentales, se observa que los 
varones que poseen un perfil agresivo impulsivo, y los varones que poseen un perfil agresivo proactivo, tienen alta Espontaneidad vital e Implicación vital, comprometiéndose más con lo que les rodea y las causas que defienden. Mientras que las mujeres con un perfil de alta agresividad impulsiva, y las mujeres con un perfil de alta agresividad proactiva, tienen alto Hiperanálisis, Afrontamiento borroso e Hipercontrol anticipatorio, siendo más reflexivas e indecisas a la hora de afrontar los retos y alarmándose de sobremanera con el futuro que les espera.

Además de lo dicho anteriormente, los varones con perfil agresivo impulsivo, tienen alta Autoconvicción volitiva, autojustificándose para convencerse a sí mismos sobre el por qué realizan determinados actos, o no. Por su parte, las mujeres con perfil agresivo proactivo, poseen más Atribución a las estrategias, ya que consideran que la manera de actuar que tienen, es el origen de sus fracasos.

\section{Relación entre rasgos de personalidady tipos de agresividad.}

Los datos obtenidos no señalan diferencias significativas en ninguna de las dimensiones globales estudiadas, independientemente del tipo de agresividad utilizado, esto contradice lo que señalaron López y López (2003), que encontraron relaciones significativas entre agresividad, Ansiedad, Independencia, Extraversión y Autocontrol. Dentro de las escalas primarias, sólo se ha encontrado relación significativa entre la escala de Tensión y los adolescentes con perfiles agresivos impulsivos, lo que indica que son más intranquilos y se sienten más presionados.

En cuanto a las Dificultades cotidianas, los adolescentes con alta agresividad proactiva y los adolescentes con alta agresividad impulsiva, tienen un alto Total de dificultades porque puntúan alto en las escalas de las que se compone, (a) Ira-agresión, (b) Dificultad con la autoridad y (c) Dificultad con la adicción. Estas dificultades señalan que los adolescentes con altos niveles de agresividad, son propensos a actuar llevados por la ira, tienen mala tolerancia a sus superiores o figuras de referencia, y sufren un descontrol en el consumo de alcohol y drogas. Además, los adolescentes con alta agresividad impulsiva presentan un alto afrontamiento deficiente, teniendo serias dificultades para comprender cómo deben enfrentar los problemas de manera eficiente.
D. Tipos de agresividad, desplazamiento emocional y disociación emocional.

Los resultados hallados al respecto, muestran que a mayor agresividad impulsiva existe mayor utilización del desplazamiento emocional, mientras que a mayor agresividad proactiva se produce un mayor empleo de la disociación emocional. Esto confirma los estudios realizados por Alcázar-Córcoles, Verdejo-García, BousoSaiz, y Bezos-Saldaña (2010), en relación a los diferentes mecanismos emocionales utilizados por los dos tipos de agresividad.

Como conclusión se establece que (a) existen diferencias en función del sexo en el nivel de agresividad, siendo mayor en mujeres, (b) que la Tensión es el rasgo de personalidad principal de los adolescentes agresivos y, (c) los encuadres de Ajuste y Espontaneidad vital son los moldes mentales más utilizados por estos adolescentes.

\section{REFERENCIAS}

Alcázar-Córcoles, M.A., Verdejo-García, A., Bouso-Saiz, J.C., y BezosSaldaña, L. (2010). Neuropsicología de la agresión impulsiva. Revista de neurología, 50, 291-299.

Alsaker, F.D. (1996). Annotation: The impact of puberty. Journal of Child Psychology and Psychiatry, 37, 249-258. doi: 10.1111/j.14697610.1996.tb01403.x.

Anderson, C., \& Bushman, B. (2002). Human Aggression. Annual Review Psychology, 53, 27-51. doi: 10.1146/annurev.psych.53.100901.135231.

Andreu, J.M. (2010). Manual: Cuestionario de agresividad premeditada e impulsiva en adolescentes. CAPI-A. Madrid: TEA Ediciones.

Archer, D. \& McDaniel, P. (1995). Violence and gender: Differences and similarities across societies. In R.B. Ruback, \& N.A Weiner (Eds.), Interpersonal Violent Behaviours: Social and Cultural Aspects (pp.63-87). New York: Springer.

Berkowitz, L. (1989). The frustration-aggression hypothesis: An examination and reformulation. Psychological Bulletin, 106, 59-73. doi: 10.1037/0033-2909.106.1.59.

Berkowitz, L. (1996). Agresión: causas, consecuencias y control. (1a ed.). Bilbao: Desclée de Brouwer.

Bettencourt, B.A., \& Kernahan, C. (1997). A meta-analysis of aggression in the presence of violent cues: Effects of gender differences and aversive provocation. Aggressive Behavior, 23, 447-456. doi: 10.1002/(SICI)1098-2337(1997)23:6<447::AID-AB4>3.0.CO;2-D.

Björkqvist, K., Lagerspetz, K., \& Österman, K. (1994). Sex differences in covert aggression among adults. Aggressive Behavior, 20, 27-33. doi: 10.1002/1098-2337(1994)20:1<27::AID-AB2480200105>3.0.CO;2Q.

Björkqvist, K., \& Niemelä, P. (1992). New trends in the study of female 
aggression. In K. Bjorkqvist, \& P. Niemelä (Eds.), Of mice and women: Aspects of female aggression. San Diego: Academic Press.

Bowlby, J. (1982). Attachment and Loss, Vol.1, Attachment (2nd ed.). New York: Basic Books.

Buss, A.H. (1961). The psychology of aggression. (1st ed.). Oxford: Wiley.

Campbell, A. (2006). Sex differences in direct aggression: What are the psychological mediators? Aggression and Violent Behavior, 11, 237-264. doi: 10.1016/j.avb.2005.09.002.

Carrasco, M.A., \& González, M.J. (2006). Aspectos conceptuales de la agresión: definición y modelos explicativos. Acción psicológica, 4(2), 7 38. doi: 10.5944/ap.4.2.478.

Crick, N.R., \& Dodge, K.A. (1996). Social information - processing mechanism in reactive and proactive aggression. Child Development, 67, 993-1002. doi: 10.1111/j.1467-8624.1996.tb01778.x

Dodge, K.A. (1991). The structure and function of reactive and proactive aggression. In D. J. Pepler \& K. H. Rubin. The development and treatment of childhood aggression (pp. 201-218). Hillsdale: Lawrence Erlbaum Associates.

Dodge, K.A., Bates, J.E., \& Petit, G.S. (1990). Mechanisms in the cycle of violence. Science, 250, 678 - 683. doi: 10.1126/science.2270481.

Dodge, K. A., \& Coie, J.D. (1987). Social information processing factors in reactive and proactive aggression in children's peer groups. Journal of Personality and Social Psychology, 53, 1146 - 1158. doi: 10.1037//0022-3514.53.6.1146.

Dodge, K.A., \& McCourt, S.N. (2010). Translating models of antisocial behavioral development into efficacious interventions policy to prevent adolescent violence. Developmental Psychobiology, 52(3), 277 285. doi: 10.1002/dev.20440.

Dollard, J., Doob, L.W., Miller, N.E., Mowrer, O.H., \& Sears, R.R. (1968). Frustration and aggression. (2nd ed.). New Haven: Yale University Press.

Farrington, D.P. (1994). Childhood, adolescent and adult features of violent males. In L.R. Huessman (Ed.), Aggressive behaviour: Current perspectives (pp. 215-240). New York: Plenum Press.

Galen, B.R., \& Underwood, M.K. (1997). A developmental investigation of social aggression among children. Developmental Psychology, 33(4), 589-600. doi: 10.1037/0012-1649.33.4.589.

Geen, R.G. (2001). Human aggressiveness. (2nd ed.). USA: Open University Press.

Hernández - Guanir, P. (2010). Manual: Test de estrategias cognitivo emocionales. MOLDES. Madrid: TEA Ediciones.
Huesman, L.R. (1988). An information processing model for the development of aggression. Aggressive Behavior, 11, 13-24. doi: 10.1002/1098-2337(1988)14:1<13::AID-AB2480140104>3.0.CO;2$\mathrm{J}$.

Liu, J. (2004). Concept analysis: Aggression. Issues in Mental Health Nursing, 25, 693-714. doi: 10.1080/016128404904486755.

López, C., \& López, J. R. (2003). Rasgos de personalidad y conducta antisocial y delictiva. Psicopatología Clínica, Legaly Forense, 3, 5-19.

Mestre, V., Samper, P., Frías, M.D., \& Nácher, M.J. (2003). Estilos de crianza y variables personales como factores de riesgo de la conducta agresiva. Revista Méxicana de Psicología, 20, 189-199.

Moffitt, T. E., \& Caspi, A. (2001). Childhood predictors differentiate life-course persistent and adolescence-limited antisocial pathways among males and females. Development and Psychopathology, 13, 355375. doi: 10.1017/S0954579401002097.

Muñoz Vivas, F. (2000). Adolescencia y agresividad. Tesis doctoral publicada, Universidad Complutense, Madrid.

Olweus, D. (1978). Aggression in the schools: Bullies and Whipping Boys. (1st ed.). New York: Wiley.

Oñate, A., \& Piñuel, I. (2006). Estudio Cisneros X. Violencia y acoso escolar en España. Madrid: Instituto de Innovación Educativa y Desarrollo Directivo.

Penado Abilleira, M. (2012). Agresividad reactiva y proactiva en adolescentes: efecto de los factores individuales y socio-contextuales. Tesis doctoral no publicada, Universidad Complutense, Madrid.

Pinillos, J.L. (1990). La adolescencia en las postrimerías de la modernidad. Psicopatología, 10, 174-176.

Scandroglio, B., Martínez, J. M., Martín, M. J., López, J. S., Martín, A., San José, M. C., \& Martín, J. M. (2002). Violencia grupal juvenil: una revisión crítica. Psicothema, 14, supl.6-15.

Schuerger, J.M. (2005). Manual: Cuestionario de Personalidad para Adolescentes 16PF-APQ. Madrid: TEA Ediciones.

Toldos, M.P. (2005). Sex And Age Differences in Self-Estimated Physical, Verbal and Indirect Aggression in Spanish Adolescents. Aggressive Behaviour, 31, 13-23. doi: 10.1002/ab.20034.

Tremblay, R.E., Boulerice, B., Harden, P.W., McDuff, P., Pérusse, D., Pihl, R.O., \& Zocolillo, M. (1996). Do children in Canada become more aggressive as they approach adolescence? In Human Resources Development Canada \& Statistics Canada (Eds.), Growing up in Canada: National Longitudinal Survey of Children and Youth (pp. 127-137). Ottawa: Statistics Canada 